\title{
Saturation and Metastable Properties of the van der Waals Fluid
}

\author{
N. SHAMSUNDAR and JOHN H. LIENHARD
}

\author{
Heat Transfer/Phase Change Laboratory, Mechanical Engineering Department, University of Houston, \\ Houston, Texas 77004
}

\begin{abstract}
Modern applications require accurate thermodynamic equations of state that portray stable and metastable states of fluids. Earlier work has shown that the famous van der Waals equation, contrary to widely held views, finds its natural niche in the rank of real substances ordered according to their values of the acentric factor. In this note, the saturated and metastable state properties of a van der Waals fluid are presented in the form of a temperature table and a pressure table. A comparison of these results with the available data for mercury is made and it is established that mercury is approximated rather well as a van der Waals fluid.
\end{abstract}

Les applications modernes exigent l'emploi d'équations d'état thermodynamiques exactes décrivant les états stable et métastable des fluides. Un travail antérieur a montré que la fameuse équation de van der Waals, contrairement à certains points de vue exprimés, trouve une place naturelle dans la classe des substances réelles ordonnées selon leurs valeurs du facteur "d'acentricité". On présente les propriétés à l'état saturé et métastable d'un fluide de van der Waals, sous forme d'un tableau de température et d'un tableau de pression. On a comparé ces résultats avec les données disponibles pour le mercure et l'on a établi que le mercure est assez bien représenté par un fluide de van der Waals.

$\mathrm{T}$ here has been strong recent interest in the formulation of equations of state, driven in part by the problems of dealing with the superheated liquids and supercooled vapors that arise in many modern heat transfer processes. Such problems arise in nuclear reactors, light hydrocarbon spills, and in the expansion of vapors in turbines. It is no longer enough to have equations that will only model stable equilibrium states. Today, there is a serious need for equations that will also represent the metastable liquid and vapor states (sometimes, although incorrectly, called non-equilibrium states.)

Most existing equations of state are inherently capable of correctly reproducing metastable states, at least qualitatively. This is particularly true of the many different algebraic equations of state whose parameters are determined with the use of very few experimental results - for example, those based on the critical quantities and a known boiling point. It is well known that many such equations are valid only for substances with a specific value of the critical compressibility, $Z_{c}$. The best known simple eq: ation of state, the van der Waals equation, gives $Z_{c}=3 / 8$. The one most often quoted by chemical engineers, the RedlichKwong equation, gives $Z_{c}=1 / 3$. This value is much closer to the $Z_{\mathrm{c}}$ of most inorganic gases and hydrocarbons; thus the Redlich-Kwong equation is better suited for these substances than is the van der Waals equation. The common inference from these - that the Redlich-Kwong equation is more accurate than the van der Waals equation - is misleading since an equation of state should only represent those substances whose $Z_{c}$ lies close to the $Z_{c}$ of the equation.

This train of thought was pursued convincingly by Peck (1982), whose compilation and analysis shows that the van der Waals substance, i.e., one with $Z_{c}=3 / 8$, finds its natural niche in the rank of real substances when they are ordered by their values of $Z_{c}$. Actually, Peck used a more effective characterization of molecular structure than $Z_{c}$, namely the Pitzer acentric factor, $\omega=-[1+\log p$
$(T=0.7)]$. ( $\omega=-0.302$ for a van der Waals fluid, or 0.058 for a Redlich-Kwong fluid.)

Peck's conclusion (which we endorse) is, in effect, that if a real substance with $Z_{c}=3 / 8$ or $\omega=-0.302$ were to be discovered, it would obey the van der Waals equation and would conform to all conclusions based upon that equation. This statement rested only upon circumstantial evidence, for we do not know of a van der Waals substance. Further evidence supporting Peck's conclusion will be given in this paper. This evidence involves data for mercury, which has a value of $Z_{c}$ close to $3 / 8$, and $\omega$ close to -0.302 .

Once it is accepted that the van der Waals substance, with mercury being a conceivable example thereof, has its place in the hierarchy of real substances, it follows that, in formulating new equations of state applicable to substances with a range of values of $Z_{c}$, the properties of the van der Waals substance should be used as constraints. It should be possible to recover the properties of the van der Waals substance by substituting $3 / 8$ for $Z_{c}$ in the new equations. Lienhard (1982) has shown that including the van der Waals equation in this way leads to improved results.

In the program we have undertaken for formulating such equations, we have often felt the need for a tabulation of the properties of a van der Waals substance. No such tabulation is available within the knowledge of the authors, although the calculations leading to such a table are, in the main, simple. Graphical presentations of this or that individual property of the van der Waals substance are scattered in numerous papers. In this paper, we present a pair of complete tables of properties of the saturated and metastable van der Waals fluid, calculated with high precision. A new analytical approximation for the vapor pressure at low temperatures is also supplied.

\section{Calculations}

All physical properties in the following dicussion are reduced quantities. 


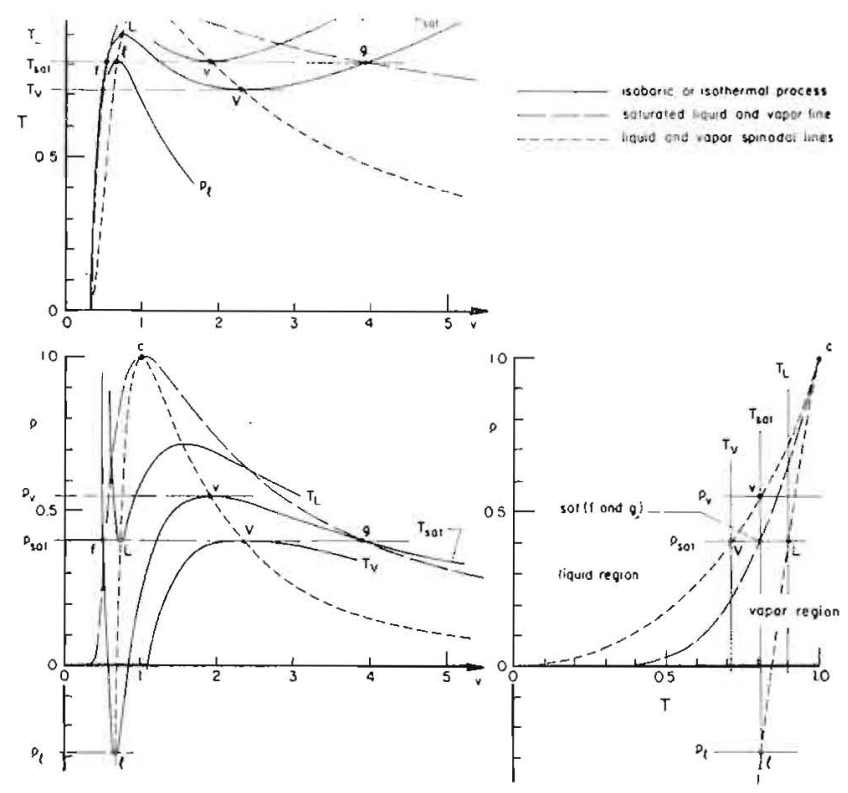

Figure 1 - van der Waals $p-v-T$ surface.

The coexistence curve is best calculated using Rowlinson's (1958) technique. The advantage of his method over the alternative procedure described by Peck (1982) is that no cubic equations have to be solved. Over a wide range of temperatures and pressures, the coefficients of the cubic equation would span several orders of magnitude, and the roots could be widely separated, calling for care in the numerical work. Rowlinson's method gives values of $T, p_{\text {sat }}$, $v_{\mathrm{f}}$ and $v_{\mathrm{g}}$ for a given value of the parameter $r \equiv\left(3 v_{\mathrm{f}}-1\right) /$ $\left(3 v_{\mathrm{g}}-1\right)$. To obtain tables with regular values of $p$ or $T$, as we desire, it is necessary to build an iteration scheme around Rowlinson's sequence of calculations. We employed the secant method.

To facilitate the calculations, we supply a set of equations that will furnish an estimate of the parameter $r$ with which the iterations are to be started:

$$
r_{\text {est }}=\left(p_{\text {sat }} / 22\right)\left(1+10 p_{\text {sat }}\right)
$$

If $T$ is the primary variable, it becomes necessary to estimate $p_{\text {sat }}$ before using this equation. The following approximations are recommended.

For $T>0.3$,

$$
\ln p_{\text {sat }} \cong 4.5267-5.1227 /(T+0.1317) \ldots \ldots
$$

For $T \leqslant 0.3$,

$$
-\ln \left(p_{\text {sat }} / 27\right) \cong 1+729 /[8 T(8 T+27)] \ldots \ldots
$$

The medium-high temperature approximation (2) is an Antoine correlation developed by Lienhard (1976). The low-temperature approximation (3) is new, and was obtained by developing series expansions of the governing equations at low pressures. The correlation (1) is also new.

Spinodal points are found by applying the condition $(\partial p / \partial v)_{\mathrm{T}}=0$ to the van der Waals equation. The result may be written in either of two forms:

$$
4 T v^{3}-(3 v-1)^{2}=0 \text { or } p v^{3}-3 v+2=0 \ldots
$$

The spinodal volumes are the two larger roots of these equations.

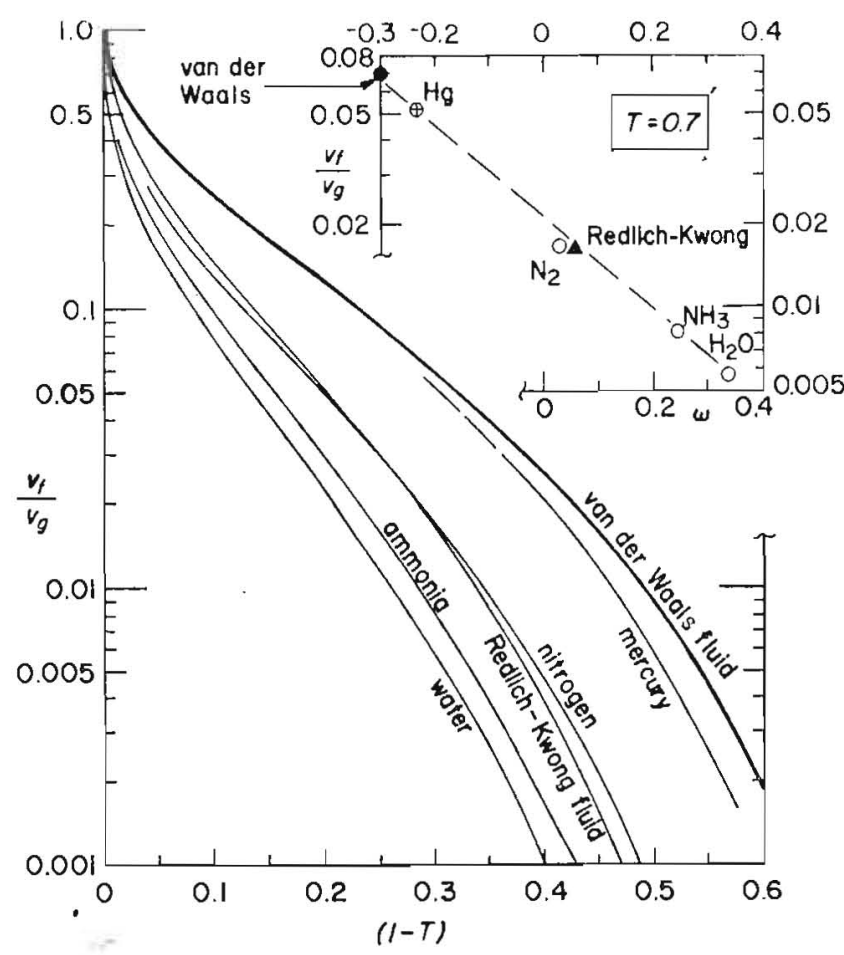

Figure 2 - Comparison of some typical $v_{\mathrm{f}} / v_{\mathrm{g}}$ curves for fluids with different Pitzer "acentric" factors.

Internal energy, enthalpy, and entropy are related to their ideal gas values (marked*) by the relations

$$
\begin{aligned}
& u^{*}-u=9 / 8 v \ldots \ldots \ldots \ldots \\
& h^{*}-h=9 / 8 v+T-(3 / 8) p v \\
& s^{*}-s=-\ln (1-3 v) \ldots \ldots
\end{aligned}
$$

The use of these equations bypasses the use of the ClausiusClapeyron equation to find $u_{\mathrm{fg}}, h_{\mathrm{fg}}$ and $s_{\mathrm{fg}}$. Thus, we avoid introducing the inaccuracy associated with finding the slope of the vapor pressure curve.

Figure 1 is used to explain the notation for the various states. With reference to the figure, the following enthalpy differences are calculated using Equation (6):

Isothermal differences:

$$
\Delta h_{l} \equiv h_{l}-h_{\mathrm{f}}, \quad \Delta h_{\mathrm{v}} \equiv h_{\mathrm{g}}-h_{\mathrm{v}}
$$

Isobaric differences:

$$
\Delta h_{\mathrm{L}} \equiv h_{\mathrm{L}}-h_{\mathrm{f}}, \quad \Delta h_{\mathrm{V}} \equiv h_{\mathrm{g}}-h_{\mathrm{V}}
$$

The availability, $\Delta a$, of a spinodal state with reference to its neighbouring saturation state is of interest because it indicates how much damage a flashing liquid could possibly accomplish. The following four availabilities are based on the reference temperature being $T_{\text {sat }}$, with $a=h-T_{\text {sal }} s$ :

Isothermal availabilities:

$$
\Delta a_{l} \equiv a_{\text {sal }}-a_{l}, \quad \Delta a_{\mathrm{v}}=a_{\mathrm{v}}-a_{\text {sat }} \ldots \ldots \ldots \ldots
$$

Isobaric availabilites:

$$
\Delta a_{\mathrm{L}} \equiv a_{\mathrm{L}}-a_{\mathrm{sal}}, \quad \Delta a_{\mathrm{v}}=a_{\mathrm{V}}-a_{\text {sat }}
$$

Here, note that $a_{\text {sat }}=a_{\mathrm{f}}=a_{\mathrm{g}}$. In calculating the isobaric differences in equations (9) and (11), we have used $c_{\mathrm{p}} / R=5 / 2$. 
Table 1

van der Waals Saturation and Spinodal Properties: Temperature Table

T

$\begin{array}{ll}1 & 1 \\ 0.98 & 0.922191 \\ 0.96 & 0.84762 \\ 0.96 & 0.77707\end{array}$

$0.94 \quad 0.77707$

$\begin{array}{ll}0.90 & 0.64700 \\ 0.88 & 0.58736\end{array}$

$\begin{array}{ll}0.88 & 0.58736 \\ 0.86 & 0.53175\end{array}$

$\begin{array}{ll}0.84 & 0.47859 \\ 0.82 & 0.42932\end{array}$

$0.80 \quad 0.38336$

$0.78 \quad 0.34084$

$\begin{array}{ll}0.76 & 0.340119 \\ 0.74 & 0.26459 \\ 0.72 & 0.23108\end{array}$

$0.70 \quad 0.20046$

$\begin{array}{ll}0.68 & 0.17262 \\ 0.66 & 0.14745\end{array}$

$\begin{array}{ll}0.64 & 0.12485 \\ 0.62 & 0.10470\end{array}$

$0.60 \quad 0.86869-1$

$0.56 \quad 0.57645-1$

$\begin{array}{ll}0.54 & 0.45975-1 \\ 0.52 & 0.36073-1\end{array}$

$0.50 \quad 0.27789-1$

$0.48 \quad 0.20967-1$

$\begin{array}{ll}0.46 & 0.15451-1 \\ 0.44 & 0.11084-1\end{array}$

$0.420 .77097-2$

$\begin{array}{ll}0.40 & 0.51745-2 \\ 0.38 & 0.33318-2\end{array}$

$0.360 .20435-2$

$\begin{array}{ll}0.32 & 0.11834-2 \\ 0.32 & 0.64005-3\end{array}$

$0.30 \quad 0.31882-3$

$0.28 \quad 0.14367-3$

$0.26 \quad 0.57229-4$

$0.220 .54768-5$

$\begin{array}{lll}0.20 & 0.11891-5 & 0.3558 \\ 0.18 & 0.18358-6 & 0.3533\end{array}$

$0.160 .17734-7 \cdot 0.35084$

$0.12 \quad 0.15878-10 \quad 0.34611$

$0.10 \quad 0.57631-13 \quad 0.34384$

$\begin{array}{lll}0.08 & 0.12561-76 & 0.34153 \\ 0.06 & 0.98727-23 & 0.33943\end{array}$ $\begin{array}{lllllllll}v_{g} & v_{f g} & h_{f g} & s_{f g} & u_{f g} & v_{l} & v_{v} & \Delta P_{l} & \Delta P_{v}\end{array}$

$\begin{array}{lccccccccc}1 & 1 & 1 & 0 & 0 & 0 & 0 & 1 & 1 & 0 \\ 0.77554 & 1.3761 & 0.0005 & 0.8407 & 0.8570 & 0.5339 & 0.85611 & 1.1892 & 0.01616 & 0.01018\end{array}$ $\begin{array}{llllllllll}0.0817 & 1.6119 & 3.9036 & 1.1778 & 1.2269 & 0.33936 & 0.85593 & 1.2867 & 0.046161 & 0.01018\end{array}$

$\begin{array}{llllllllllll}0.63022 & 2.0369 & 1.4567 & 1.6340 & 1.77760 & 1.2460 & 0.74221 & 1.4511 & 0.15591 & 0.05903\end{array}$

$\begin{array}{llllllllll}0.65343 & 2.3439 & 1.7454 & 1.8090 & 2.0100 & 1.3855 & 0.71863 & 1.5285 & 0.22716 & 0.07701\end{array}$ $\begin{array}{llllllllll}0.58105 & 2.6360 & 2.0547 & 1.9619 & 2.2295 & 1.5073 & 0.69814 & 1.5052 & 0.30985 & 0.077340\end{array}$

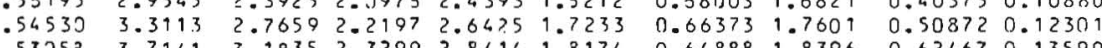
$\begin{array}{llllllllllll}0.51741 & 4.1725 & 3.6550 & 2.4301 & 3.0377 & 1.7047 & 0.63521 & 1.9212 & 0.75155 & 0.14737\end{array}$

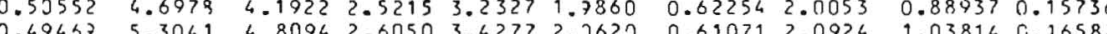
$0.48479 \quad 0.009$ ? $5.5244 \quad 2.5816 \quad 3.6238231334 \quad 0.59960 \quad 3.1828 \quad 1.19793 \quad 0.17274$ $\begin{array}{llllllllll} & 0.8354 & 5.3597 & 2.7517 & 3.8218 & 2.2036 & 0.58912 & 2.2770 & 1.36881 & 0.17812\end{array}$

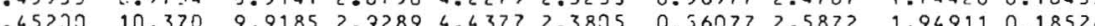
$\begin{array}{llllllll} & 0.45230\end{array}$

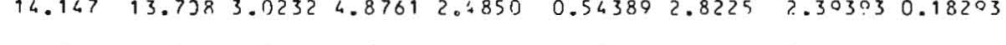

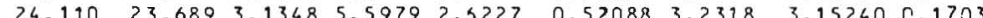

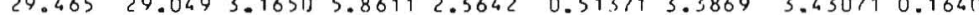
$0.40675 \quad 45.984 \quad 5.57733 .2163 \quad 6.4326 \quad 2.74730 .50000 \quad 3.7321 \quad 4.037790 .14013$ $\begin{array}{lllllllllll}5 & 5.9969 & 53.567 & 3.2377 & 0.7453 & 2.7772 & 0.49342 & 3.9252 & 4.34748 & 0.14068\end{array}$

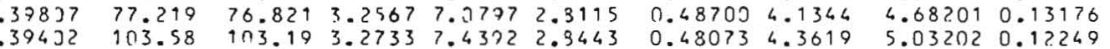

$\begin{array}{llllllllll}0.38641 & 203.63 & 203.24 & 3.3003 & 8.2507 & 2.7059 & 0.46857 & 4.8833 & 5.78159 & 0.10346\end{array}$

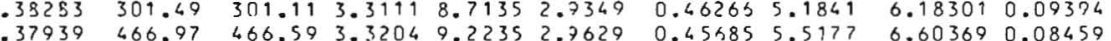

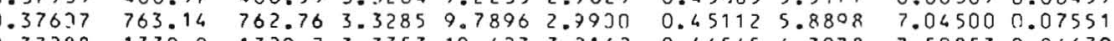

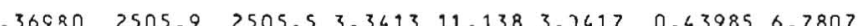
$5193.25192 .93 .346411 .952 \quad 3.3667 \quad 0.434337 .32068$

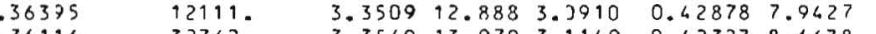
0.10711 .06

$0.44352+06$

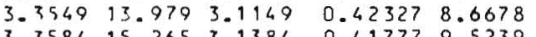

7.996200 .05851 8.510250 .05074 9.053410 .04350

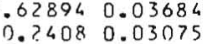
$0.44852+06$
$0.26147+07$
$0.24359+08$ $0.26359+08$ $0.20153+11$ $\begin{array}{lllll}3.3615 & 16.807 & 3.1615 & 0.41225 & 10.550 \\ 3.3642 & 18.690 & 3.1842 & 0.40669 & 11.804\end{array}$ 18.6903 .1842 0.40669
0.40103
0.31 .804 $\begin{array}{ll}0.8941 & 0.02525 \\ .5948 & 0.02031\end{array}$ $\begin{array}{ll}1.5948 & 0.02031 \\ 2.35122 & 0.01595\end{array}$ $\begin{array}{lllllll}3.3687 & 24.062 & 3.2287 & 0.39534 & 13.382 & 13.1738 & 0.01213\end{array}$

$\begin{array}{llllll}0.46271+13 & 3.3718 & 33.718 & 3.2718 & 0.38338 & 21.818\end{array}$

$0.16205+23$
$\Delta h_{l} \quad \Delta h_{v} \quad \Delta a_{l} \quad \Delta a_{v}$

$\begin{array}{cccc}0 & 0 & 0 & 0 \\ 0.15919 & 0.18851 & 0.00484 & 0.00501\end{array}$ $\begin{array}{llll}0.25875 & 0.26736 & 1.01367 & 0.01133 \\ 0.23799 & 0.32587 & 0.02571 & 0.0266\end{array}$ $\begin{array}{lllll}0.75577 & 0.37268 & 0.03868 & 0.04137\end{array}$

$\begin{array}{lllll}0.26562 & 0.41194 & 0.05411 & 0.05838\end{array}$ $\begin{array}{llll}0.26937 & 0.44470 & 0.07124 & 0.058386 \\ 0.26817 & 0.47238 & 0.08993 & 0.09852\end{array}$

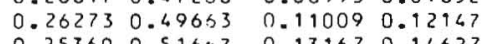
$\begin{array}{lllll}0.24114 & 0.53340 & 0.15460 & 0.17287\end{array}$

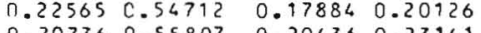
$\begin{array}{llll}0.26736 & 0.55877 & 0.20436 & 0.2334 \\ 0.18643 & 0.56644 & 0.23113 & 0.26333 \\ 0.16302 & 0.57239 & 0.25914 & 0.29702\end{array}$ $\begin{array}{lllll}0.13724 & 0.57606 & 0.28836 & 0.33269\end{array}$ $\begin{array}{lllll}0.07990 & 0.57700 & 0.35046 & 0.40879\end{array}$ $0.04647 \quad 0.57447 \quad 0.383320 .44968$ $0.011940 .57006 \quad 0.41740 \quad 0.49243$ $\begin{array}{llllll}-0.02467 & 0.56385 & 0.45271 & 0.53708\end{array}$

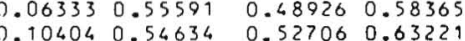
$\begin{array}{lllll}-0.14678 & 0.535 ? 0 & 0.56616 & 0.68279\end{array}$ $\begin{array}{llll}-0.19157 & 0.52258 & 0.60657 & 0.73544\end{array}$ $\begin{array}{lllll}0.23844 & 0.50857 & 0.64833 & 0.79024\end{array}$ $\begin{array}{lllll}-0.33849 & 0.47633 & 0.73611 & 0.90651\end{array}$ $\begin{array}{llll}-0.39178 & 0.45712 & 0.78224 & 0.96813 \\ -0.44733 & 0.44052 & 0.82997 & 1.03218\end{array}$ $\begin{array}{lllll}-0.50523 & 0.421 .96 & 0.87937 & 1.09876\end{array}$ $\begin{array}{llll}-0.56558 & 0.40056 & 0.93055 & 1.16796 \\ -0.62850 & 0.33057 & 0.98365 & 1.23989\end{array}$ $\begin{array}{rlll}-0.52850 & 0.38077 & 0.98365 & 1.23989 \\ -0.09615 & 0.35832 & 1.03880 & 1.31667\end{array}$ $\begin{array}{llll}-0.69415 & 0.35832 & 1.03880 & 1.31467 \\ -0.76271 & 0.33724 & 1.09617 & 1.39243\end{array}$ $\begin{array}{llll}-0.933441 & 0.31545 & 1.15599 & 1.47332\end{array}$ $\begin{array}{llll}-0.90951 & 0.29358 & 1.21849 & 1.55749\end{array}$ $\begin{array}{llll}-0.98833 & 0.27171 & 1.288398 & 1.04513 \\ -1.07128 & 0.24992 & 1.35283 & 1.73646\end{array}$

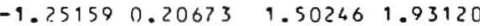

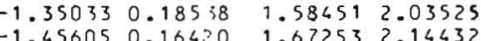
$-1.57005 \quad 0.14317 \quad 1.767792 .25896$ $\begin{array}{lllll}-1.83096 & 0.10158 & 1.98703 & 2.50799\end{array}$ $\begin{array}{llll}-2.16157 & 0.086055 & 2.11731 & 2.06469 \\ -2.26890 & 2.79200\end{array}$ 
Table 2

van der Waals Saturation and Spinodal Properties: Pressure Table

\begin{tabular}{|c|c|c|c|c|c|c|c|c|c|c|c|c|c|c|c|}
\hline P & $T_{\text {sot }}$ & $v_{f}$ & $v_{9}$ & $v_{f g}$ & $h_{f g}$ & $s_{f g}$ & $u_{p g}$ & $v_{L}$ & $v_{v}$ & $\Delta T_{L}$ & $\Delta T_{v}$ & $\Delta h_{L}$ & $\Delta h_{v}$ & $\Delta a_{L}$ & $\Delta q_{v}$ \\
\hline $\begin{array}{l}1 \\
0.98 \\
0.96 \\
0.96 \\
0.92\end{array}$ & $\begin{array}{c}1 \\
0.99497 \\
0.98988 \\
0.98472 \\
0.97950\end{array}$ & $\begin{array}{c}1 \\
0.87437 \\
0.83004 \\
0.79848 \\
0.77333\end{array}$ & $\begin{array}{c}1 \\
1.1623 \\
1.2448 \\
1.3159 \\
1.3823\end{array}$ & $\begin{array}{c}0 \\
0.2979 \\
0.4147 \\
0.5175 \\
0.6390\end{array}$ & $\begin{array}{c}0 \\
0.6266 \\
0.5009 \\
0.7364 \\
0.8510\end{array}$ & $\begin{array}{c}0 \\
0.4267 \\
0.6070 \\
0.7479 \\
0.8688\end{array}$ & $\begin{array}{c}0 \\
0.3137 \\
0.4516 \\
0.5540 \\
0.0439\end{array}$ & $\begin{array}{c}1 \\
0.92630 \\
0.89776 \\
0.88072 \\
0.86547\end{array}$ & $\begin{array}{c}1 \\
1.0917 \\
1.1369 \\
1.1751 \\
1.2902\end{array}$ & $\begin{array}{c}0 \\
0.00041 \\
0.00116 \\
0.00214 \\
0.00332\end{array}$ & $\begin{array}{c}0 \\
0.00643 \\
0.00199 \\
0.00220 \\
0.00343\end{array}$ & $\begin{array}{c}0 \\
0.0918 \\
0.1319 \\
0.1638 \\
0.1916\end{array}$ & $\begin{array}{c}0 \\
0.0897 \\
0.1264 \\
0.1554 \\
0.1303\end{array}$ & $\begin{array}{c}0 \\
0.00003 \\
0.00010 \\
0.00024 \\
0.03046\end{array}$ & $\begin{array}{c}0 \\
0.00003 \\
0.00010 \\
0.00024 \\
0.00043\end{array}$ \\
\hline $\begin{array}{l}0.90 \\
0.88 \\
0.86 \\
0.84 \\
0.82\end{array}$ & $\begin{array}{l}0.97421 \\
0.96884 \\
0.96340 \\
0.95789 \\
0.95230\end{array}$ & $\begin{array}{l}0.75218 \\
0.73380 \\
0.71748 \\
0.70274 \\
0.68928\end{array}$ & $\begin{array}{l}1.4664 \\
1.5056 \\
1.5726 \\
1.0361 \\
1.7005\end{array}$ & $\begin{array}{l}0.6942 \\
0.7758 \\
0.8559 \\
0.9334 \\
1.0113\end{array}$ & $\begin{array}{l}0.9522 \\
1.0639 \\
1.1284 \\
1.2073 \\
1.2815\end{array}$ & $\begin{array}{l}0.9776 \\
1.0774 \\
1.9712 \\
1.2603 \\
1.3657\end{array}$ & $\begin{array}{l}0.7179 \\
0.7879 \\
0.8526 \\
0.9133 \\
0.9736\end{array}$ & $\begin{array}{l}0.85260 \\
0.84139 \\
0.83142 \\
0.82242 \\
0.81620\end{array}$ & $\begin{array}{l}1.2635 \\
1.2758 \\
1.3076 \\
1.3393 \\
1.3709\end{array}$ & $\begin{array}{l}0.00467 \\
0.00818 \\
0.00785 \\
0.00960 \\
0.01162\end{array}$ & $\begin{array}{l}0.00684 \\
0.00042 \\
0.00817 \\
0.01008 \\
0.01215\end{array}$ & $\begin{array}{l}0.2171 \\
0.2608 \\
0.2634 \\
0.2852 \\
0.3062\end{array}$ & $\begin{array}{l}0.2027 \\
0.2233 \\
0.2626 \\
0.2610 \\
0.2786\end{array}$ & $\begin{array}{l}0.00070 \\
0.00103 \\
0.00143 \\
0.00191 \\
0.01247\end{array}$ & $\begin{array}{l}0.00068 \\
0.00100 \\
0.00140 \\
0.00186 \\
0.00249\end{array}$ \\
\hline $\begin{array}{l}0.80 \\
0.78 \\
0.76 \\
0.74 \\
0.72\end{array}$ & $\begin{array}{l}0.96662 \\
0.94085 \\
0.93500 \\
0.92905 \\
0.92300\end{array}$ & $\begin{array}{l}0.67686 \\
0.66532 \\
0.65452 \\
0.64435 \\
0.63474\end{array}$ & $\begin{array}{l}1.7663 \\
1.8338 \\
1.9032 \\
1.9750 \\
2.0494\end{array}$ & $\begin{array}{l}1.0895 \\
1.1585 \\
1.2687 \\
1.3307 \\
1.4947\end{array}$ & $\begin{array}{l}1.3520 \\
1.4192 \\
1.4836 \\
1.5456 \\
1.6054\end{array}$ & $\begin{array}{l}1.4282 \\
1.5084 \\
1.5868 \\
1.6636 \\
1.7393\end{array}$ & $\begin{array}{l}1.0252 \\
1.0774 \\
1.1277 \\
1.1763 \\
1.2234\end{array}$ & $\begin{array}{l}0.30661 \\
0.79957 \\
0.79300 \\
0.78682 \\
0.78100\end{array}$ & $\begin{array}{l}1.4028 \\
1.4351 \\
1.4078 \\
1.5013 \\
1.5354\end{array}$ & $\begin{array}{l}0.01372 \\
0.01596 \\
0.01834 \\
0.02087 \\
0.02354\end{array}$ & $\begin{array}{l}0.01437 \\
0.01675 \\
0.01928 \\
0.02196 \\
0.02480\end{array}$ & $\begin{array}{l}0.3209 \\
0.3479 \\
0.3679 \\
0.3870 \\
0.4067\end{array}$ & $\begin{array}{l}0.2957 \\
0.3122 \\
0.3284 \\
0.3442 \\
0.3597\end{array}$ & $\begin{array}{l}0.04312 \\
0.00387 \\
0.00479 \\
0.00566 \\
0.00572\end{array}$ & $\begin{array}{l}0.00304 \\
0.00376 \\
0.00458 \\
0.00559 \\
0.00654\end{array}$ \\
\hline $\begin{array}{l}0.70 \\
0.68 \\
0.66 \\
0.64 \\
0.62\end{array}$ & $\begin{array}{l}0.91684 \\
0.91058 \\
0.90621 \\
0.89771 \\
0.89109\end{array}$ & $\begin{array}{l}0.62562 \\
0.51693 \\
0.60862 \\
0.60065 \\
0.59299\end{array}$ & $\begin{array}{l}2.1268 \\
2.2075 \\
2.2918 \\
2.3803 \\
2.4732\end{array}$ & $\begin{array}{l}1.5312 \\
1.5706 \\
1.6832 \\
1.7760 \\
1.8903\end{array}$ & & $\begin{array}{l}1.8142 \\
1.8884 \\
1.9621 \\
2.0357 \\
2.1092\end{array}$ & $\begin{array}{l}1.2672 \\
1.3139 \\
1.3576 \\
1.4003 \\
1.4423\end{array}$ & $\begin{array}{l}0.77548 \\
0.77025 \\
0.76526 \\
0.76050 \\
0.75594\end{array}$ & $\begin{array}{l}1.5705 \\
1.6066 \\
1.6438 \\
1.6822 \\
1.7221\end{array}$ & $\begin{array}{l}0.02636 \\
0.02932 \\
0.03244 \\
0.03571 \\
0.03915\end{array}$ & $\begin{array}{l}0.02779 \\
0.03095 \\
0.03426 \\
0.03774 \\
0.04138\end{array}$ & $\begin{array}{l}0.4264 \\
0.4461 \\
0.4658 \\
0.4856 \\
0.5056\end{array}$ & & $\begin{array}{l}0.04791 \\
0.00923 \\
0.01068 \\
0.01229 \\
0.01406\end{array}$ & $\begin{array}{l}0.00770 \\
0.00898 \\
0.01040 \\
0.01197 \\
0.01377\end{array}$ \\
\hline $\begin{array}{l}0.60 \\
0.58 \\
0.56 \\
0.54 \\
0.52\end{array}$ & $\begin{array}{l}0.88434 \\
0.87744 \\
0.87040 \\
0.86320 \\
0.85584\end{array}$ & $\begin{array}{l}0.58560 \\
0.57866 \\
0.57154 \\
0.56483 \\
0.55831\end{array}$ & $\begin{array}{l}2.5712 \\
2.6748 \\
2.7845 \\
2.9031 \\
3.0254\end{array}$ & $\begin{array}{l}1.9356 \\
2.0963 \\
2.2130 \\
2.3363 \\
2.4671\end{array}$ & & & $\begin{array}{l}1.4836 \\
1.5262 \\
1.5643 \\
1.0040 \\
1.6432\end{array}$ & & $\begin{array}{l}1.7635 \\
1.8086 \\
1.8516 \\
1.8986 \\
1.0480\end{array}$ & & $\begin{array}{l}0.04520 \\
0.04979 \\
0.05336 \\
0.05773 \\
0.06228\end{array}$ & & & $\begin{array}{l}0.01600 \\
0.01813 \\
0.02046 \\
0.02301 \\
0.02580\end{array}$ & $\begin{array}{l}0.01569 \\
0.01771 \\
0.02001 \\
0.02253 \\
0.02530\end{array}$ \\
\hline $\begin{array}{l}0.50 \\
0.48 \\
0.46 \\
0.46 \\
0.42\end{array}$ & $\begin{array}{l}0.84829 \\
0.84055 \\
0.83261 \\
0.82445 \\
0.81606\end{array}$ & $\begin{array}{l}0.55195 \\
0.54574 \\
0.53966 \\
0.53371 \\
0.52787\end{array}$ & $\begin{array}{l}3.1583 \\
3.3008 \\
3.4562 \\
3.6200 \\
3.7997\end{array}$ & $\begin{array}{l}2.6063 \\
2.7551 \\
2.9166 \\
3.0862 \\
3.2719\end{array}$ & $\begin{array}{l}2.1707 \\
2.2165 \\
2.2517 \\
2.3063 \\
2.3504\end{array}$ & $\begin{array}{l}2.5589 \\
2.0370 \\
2.7164 \\
2.7974 \\
2.8802\end{array}$ & $\begin{array}{l}1.6320 \\
1.7236 \\
1.7539 \\
1.7971 \\
1.8351\end{array}$ & & $\begin{array}{l}2.0000 \\
2.0548 \\
2.1128 \\
2.1743 \\
2.2398\end{array}$ & $\begin{array}{l}0.06349 \\
0.06825 \\
0.07324 \\
0.07847 \\
0.08396\end{array}$ & $\begin{array}{l}0.06704 \\
0.07201 \\
0.07719 \\
0.08261 \\
0.08826\end{array}$ & $\begin{array}{l}0.6305 \\
0.6525 \\
0.6750 \\
0.6981 \\
0.7217\end{array}$ & & & $\begin{array}{l}0.02834 \\
0.03166 \\
0.03539 \\
0.03932 \\
0.06372\end{array}$ \\
\hline $\begin{array}{l}0.40 \\
0.38 \\
0.36 \\
0.34 \\
0.32\end{array}$ & $\begin{array}{l}0.80741 \\
0.79848 \\
0.78925 \\
0.77969 \\
0.76976\end{array}$ & $\begin{array}{l}0.52213 \\
0.59646 \\
0.51087 \\
0.50534 \\
0.69985\end{array}$ & $\begin{array}{l}3.9956 \\
4.2099 \\
4.4457 \\
4.7066 \\
4.7970\end{array}$ & $\begin{array}{l}3.4734 \\
3.6934 \\
3.9348 \\
4.2512 \\
4.2972\end{array}$ & $\begin{array}{l}2.3941 \\
2.4374 \\
2.4863 \\
2.5229 \\
2.5652\end{array}$ & $\begin{array}{l}2.9652 \\
3.0525 \\
3.1226 \\
3.2357 \\
3.3325\end{array}$ & $\begin{array}{l}1.8731 \\
1.9111 \\
1.9491 \\
1.9872 \\
2.0255\end{array}$ & $\begin{array}{l}0.71551 \\
0.71248 \\
0.70953 \\
0.70666 \\
0.70386\end{array}$ & $\begin{array}{l}2.3098 \\
2.3849 \\
2.4658 \\
2.5534 \\
2.6486\end{array}$ & & $\begin{array}{l}0.09497 \\
0.10034 \\
0.10080 \\
0.11356 \\
0.12063\end{array}$ & $\begin{array}{l}0.7460 \\
0.7709 \\
0.7967 \\
0.8233 \\
0.8510\end{array}$ & $\begin{array}{l}0.5990 \\
0.6151 \\
0.6307 \\
0.6464 \\
0.662 \%\end{array}$ & & $\begin{array}{l}0.04856 \\
0.05389 \\
0.05977 \\
0.06627 \\
0.07368\end{array}$ \\
\hline $\begin{array}{l}0.30 \\
0.28 \\
0.26 \\
0.26 \\
0.22\end{array}$ & $\begin{array}{l}0.75943 \\
0.74865 \\
0.73736 \\
0.72550 \\
0.71297\end{array}$ & $\begin{array}{l}0.49640 \\
0.48896 \\
0.48353 \\
0.47808 \\
0.67260\end{array}$ & $\begin{array}{l}5.3227 \\
5.6909 \\
6.1108 \\
6.5948 \\
7.1594\end{array}$ & $\begin{array}{l}4.8283 \\
5.2319 \\
5.6273 \\
6.1167 \\
6.0868\end{array}$ & & & & & & & & $\begin{array}{l}0.8797 \\
0.9097 \\
0.9410 \\
0.9740 \\
1.0089\end{array}$ & $\begin{array}{l}0.6785 \\
0.6948 \\
0.7114 \\
0.7281 \\
0.7451\end{array}$ & & \\
\hline $\begin{array}{l}0.20 \\
0.18 \\
0.16 \\
0.14 \\
0.12\end{array}$ & $\begin{array}{l}0.69969 \\
0.68550 \\
0.67023 \\
0.65364 \\
0.63539\end{array}$ & $\begin{array}{l}0.66707 \\
0.46144 \\
0.45569 \\
0.66977 \\
0.44361\end{array}$ & $\begin{array}{l}7.8278 \\
8.6328 \\
9.6229 \\
10.873 \\
12.503\end{array}$ & $\begin{array}{l}7.3608 \\
8.1713 \\
9.1672 \\
10.424 \\
12.364\end{array}$ & $\begin{array}{l}2.8170 \\
2.8593 \\
2.9019 \\
2.9451 \\
2.9889\end{array}$ & $\begin{array}{l}4.0261 \\
4.1711 \\
4.3297 \\
4.5056 \\
4.7041\end{array}$ & $\begin{array}{l}2.2649 \\
2.3077 \\
2.3519 \\
2.3978 \\
2.4451\end{array}$ & $\begin{array}{l}0.68842 \\
0.68604 \\
0.68371 \\
0.68143 \\
0.67020\end{array}$ & $\begin{array}{l}3.4820 \\
3.6960 \\
3.9476 \\
4.2506 \\
4.6257\end{array}$ & $\begin{array}{l}0.16985 \\
0.18139 \\
0.19402 \\
0.20798 \\
0.22363\end{array}$ & $\begin{array}{l}0.17138 \\
0.18159 \\
0.19246 \\
0.20407 \\
0.21656\end{array}$ & $\begin{array}{l}1.0459 \\
1.0854 \\
1.1281 \\
1.1745 \\
1.2257\end{array}$ & $\begin{array}{l}0.7623 \\
0.7797 \\
0.7973 \\
0.8150 \\
0.8328\end{array}$ & $\begin{array}{l}0.13450 \\
0.14977 \\
0.16733 \\
0.18779 \\
0.21200\end{array}$ & $\begin{array}{l}0.13922 \\
0.15608 \\
0.17572 \\
0.19892 \\
0.22682\end{array}$ \\
\hline $\begin{array}{l}0.90 \\
0.08 \\
0.06 \\
0.04 \\
0.02\end{array}$ & $\begin{array}{l}0.61497 \\
0.59155 \\
0.56369 \\
0.52835 \\
0.47680\end{array}$ & $\begin{array}{l}0.43712 \\
0.43015 \\
0.42242 \\
0.41362 \\
0.40162\end{array}$ & $\begin{array}{l}14.744 \\
18.008 \\
23.268 \\
33.329 \\
61.480\end{array}$ & $\begin{array}{l}14.307 \\
17.578 \\
22.845 \\
32.915 \\
61.078\end{array}$ & $\begin{array}{l}3.0339 \\
3.3802 \\
3.1289 \\
3.1812 \\
3.2409\end{array}$ & $\begin{array}{l}4.9334 \\
5.2071 \\
5.5507 \\
6.0210 \\
6.7973\end{array}$ & $\begin{array}{l}2.4974 \\
2.5529 \\
2.6148 \\
2.6874 \\
2.7828\end{array}$ & $\begin{array}{l}0.67701 \\
0.67436 \\
0.67276 \\
0.67069 \\
0.66866\end{array}$ & & $\begin{array}{l}0.24147 \\
0.26232 \\
0.28763 \\
0.32043 \\
0.36946\end{array}$ & $\begin{array}{l}0.23005 \\
0.24474 \\
0.26088 \\
0.27875 \\
0.29816\end{array}$ & $\begin{array}{l}1.2831 \\
1.3492 \\
1.4281 \\
1.5283 \\
1.6749\end{array}$ & $\begin{array}{l}0.8504 \\
0.8675 \\
0.8832 \\
0.3952 \\
0.8953\end{array}$ & $\begin{array}{l}0.24128 \\
0.27779 \\
0.32547 \\
0.39287 \\
0.50578\end{array}$ & $\begin{array}{l}0.26119 \\
0.30499 \\
0.36372 \\
0.44961 \\
0.60075\end{array}$ \\
\hline 0.01 & $\begin{array}{c}0.43416 \\
0\end{array}$ & $\begin{array}{c}0.39286 \\
1 / 3\end{array}$ & 113.47 & 113.08 & 2718 & $\begin{array}{c}7.5498 \\
\infty\end{array}$ & $\begin{array}{c}2.8537 \\
2718\end{array}$ & $2 / 3$ & $\infty^{16.977}$ & $\begin{array}{r}0.41086 \\
27 / 32\end{array}$ & $\begin{array}{c}0.30876 \\
0\end{array}$ & $\begin{array}{c}17959 \\
2718\end{array}$ & $\begin{array}{c}0.8783 \\
0\end{array}$ & $\begin{array}{c}0.61306 \\
27 / 8\end{array}$ & $\begin{array}{c}0.75224 \\
0\end{array}$ \\
\hline
\end{tabular}


and pressure differences are useful

Isobaric differences:

$$
\Delta T_{\mathrm{L}} \equiv T_{\mathrm{L}}-T_{\text {sal }}, \quad \Delta T_{\mathrm{V}} \equiv T_{\text {sat }}-T_{\mathrm{V}} \ldots \ldots \ldots
$$

Isothermal differences:

$$
\Delta p_{l} \equiv p_{\text {sat }}-p_{l}, \quad \Delta p_{\mathrm{v}} \equiv p_{\mathrm{v}}-p_{\text {sal }}
$$

\section{Results and discussion}

The results of the calculation are displayed in Table 1, a temperature table, and Table 2 , a pressure table. To complete the tables, the limiting values at $T=0$ (or $p=0$ ) were calculated analytically. These limits are shown at the foot of each table.

To enable the reader to assess the practical usefulness of the results in the tables, the results will be compared to "data" for mercury (Vargaftik 1975 and Reynolds, 1979). Mercury is the only known substance whose estimated value of $Z_{c}$ is close to the van der Waals value of $3 / 8$. Now, we do not have complete and accurate data for mercury. All the liquid metals have high critical temperatures, some of which have only been estimated. The numbers stated for $T_{\mathrm{c}}$ by various authorities show some scatter. There is much more uncertainty regarding the critical pressures and critical volumes of the liquid metals. Therefore, we have chosen a plot for comparing the results to the data which uses ony the critical temperature and avoids using the uncertain critical pressure. This is a plot of the ratio $v_{\mathrm{f}} / v_{\mathrm{g}}$ against reduced temperature. This correlation may not be as widely known as the vapor-pressure curve, the Cailletet-Mathias plot (of the mean of the reduced densities of liquid and vapor against reduced temperature), etc., but it does have the advantage stated. A set of such curves was correlated as a function of $\omega$ in Lienhard (1982).

Six such curves are shown in Figure 2. The saturation data available to us for mercury stop at a temperature far short of the critical temperature. The van der Waals results, extracted from Table 1, are also shown. The closeness of the two curves, and their considerable separation from the curves for various other substances, based on the tables of Reynolds (1979), makes us expect that mercury, is indeed, close to being a van der Waals substance. To show how the ratio $v_{\mathrm{f}} / v_{\mathrm{g}}$ varies over the spectrum of substances at any given temperature, a plot of the ratio against the Pitzer factor for $T=0.7$ is shown in the inset in Figure 2. It is clear from the inset that the van der Waals equation is a legitimate member of the family of real fluids and mercury is very close to it in behavior.

Even the saturation tables of mercury and other liquid metals are incomplete. There is no liquid superheat data for any of these, and their critical constants are not accurately known. Nevertheless, the comparison made above leads to the surmise that mercury, at least, might be approximated rather well as a van der Waals fluid.

\section{Conclusions}

Tables of saturation and spinodal thermodynamic properties of the van der Waals fluid have been made available. mercury is quite close to being a van der Waals fluid.

\section{Acknowledgements}

This work was done in conjunction with contract RP 1438-2 with the Electric Power Research Institute, Palo Alto, California. (Project Manager: G. Srikantiah)

\section{Nomenclature}

$h \quad=$ specific enthalpy $\div \mathrm{RT}_{\mathrm{c}}$

$p \quad=$ absolute pressure $\div p_{\mathrm{c}}$

$p_{\mathrm{c}} \quad=$ critical pressure $(\mathrm{Pa})$

$R=$ ideal gas constant $(\mathrm{J} / \mathrm{kg}$-mole- $\mathrm{K})$

$s=$ specific entropy $\div \mathrm{R}$

$T=$ absolute temperature $\div T_{\mathrm{c}}$

$T_{\mathrm{c}}=$ critical temperature $(\mathrm{K})$

$u=$ specific internal energy $\div \mathrm{RT}_{\mathrm{c}}$

$v=$ specific volume $\div v_{c}$

$v_{\mathrm{c}} \quad=$ critical volume $\left(\mathrm{m}^{3} / \mathrm{kg}\right)$

$Z_{\mathrm{c}}=p_{\mathrm{c}} v_{\mathrm{c}} / \mathrm{RT}_{\mathrm{c}}$

$\omega=$ Pitzer factor, $-[1+\log p(T=0.7)]$

$\Delta \mathrm{a}=$ specific availability $\div \mathrm{RT}_{\mathrm{c}}$ (see Equations (10) and (11))

$\Delta h \quad=$ see definitions in Equations (8) and (9)

$\Delta p=$ see definitions in Equation (13)

$\Delta T=$ see definitions in Equation (12)

\section{Subscripts}

$\mathrm{f}, \mathrm{g}=$ saturated liquid and vapor states respectively

$\mathrm{fg}=$ change of a property from saturated liquid to saturated vapor state at the same $T_{\text {sat }}$ and $p_{\text {sat }}$

$l, \mathrm{~L}=$ liquid spinodal points defined in Figure 1

sat $=$ saturation pressure or temperature

$\mathrm{v}, \mathrm{V}=$ vapor spinodal points defined in Figure 1

\section{Superscript}

* $\quad=$ ideal gas

\section{References}

J. H. Lienhard, "Relations Between van der Waals' Fluid and Real Substances", Iranian J. Sci. and Tech., Vol. 5, 111-116, 1976.

J. H. Lienhard, "Corresponding States Correlations of the Spinodal and Homogeneous Nucleation Limits", ASME, J. Heat Trans. 104(2), 379-38! (1982).

R. E. Peck, "The Assimilation of van der Waals Equation in the Corresponding States Family", Can. J. Chem. Eng., 60, 446- 449 (1982).

W. C. Reynolds, Thermodynamic Properties in Sl, Mech. Engr. Dept., Stanford University (1979).

J. S. Rowlinson, "The Properties of Real Gases" in Encyclopedia of Physics, ed. by S. Flügge, 7, 52, Springer-Verlag, Berlin (1958).

N. B. Vargaftik, Tables of Thermophysical Properties of Liquids and Gases, 2nd. edition, Hemisphere Publ. Corp., Washington, D.C. (1975).

Manuscript received November 5, 1982; revised manuscript received July 28, 1983; accepted for publication August 10, 1983. 